\title{
The heterogeneous age-mixing model of estimating the covid cases of different local government units in the National Capital Region, Philippines
}

\author{
Ricardo Laurio Dizon \\ Polytechnic University of the Philippines, Philippines
}

\section{A R T I C L E I N F O}

\section{Keywords:}

Covid-19

Mobility

Quarantine

Susceptible-infected-recovery model

Mitigation

Heterogenous-mixing patterns

\begin{abstract}
A B S T R A C T
Objectives: The objective of the study is to investigate the effects of transmission control efforts of the Philippine Government through implementation of Enhanced Community Quarantine (ECQ) on curtailing the exponential growth of the Corona Virus (Covid-19). Also, this study provides forecast of transmission rate of Covid-19 incorporating the heterogeneous characteristics of the group of population according to age.

Methods: The heterogeneous-mixing model was used to forecast the transmission rate of Covid-19 and effect of Enhanced Community Quarantine in various Local Government Units (LGUs) in the National Capital Region (NCR), Philippines.

Results: The result of the study revealed that the mobility of people has significant contribution to the transmission rate of Covid-19. The result of the study revealed that the mobilization of workers, who are exempted in ECQ, would greatly affect the transmission of disease to those who are in the senior citizen group. The increase in the confirmed cases of Covid-19 is associated with the transmission from those workers, who are exempted from ECQ to senior citizens and the young age group. LGUs in the NCR, Philippines will experience a different pattern of peak periods ranging from the third week of April to the last week of May.

Conclusions: This study concluded that in order to achieve the containment of the spread of Covid-19 in the Philippines, the government should be stricter in implementation of the ECQ and incorporate the different pattern of transmission of Covid-19 in deciding whether to extend or to lift the ECQ.
\end{abstract}

\section{Introduction}

The world is currently experiencing a global pandemic crisis brought about by the Corona Virus 2019 or commonly known as COVID-19. The COVID-19 is a cluster of pneumonia cases caused by the newly identified $\beta$-coronavirus, which was initially infected a huge number people living in Wuhan, China in December 2019. ${ }^{1}$ The COVID-2019 (SARS-CoV-2) is previously known as 2019-nCov may have been transmitted to people from snakes which have been caught from bats. This viruses causes a respiratory infections to human ranging from common cold to a more severe respiratory diseases. ${ }^{1,2}$

As of April 1, 2020, the global number of confirmed Covid-19 cases is 871,985 with 43,261 fatalities. ${ }^{2}$ The looming number of Covid-19 posed several countries such as China, United Kingdom, United States, Italy, and many other countries around the world to implement the concept of "locked down" system.

The locked down system aims to reduce the reproduction of the Covid-19 through maintaining social distancing. ${ }^{3}$ Pellis et al. ${ }^{3}$ emphasized the two major objectives of locked down system which are: (1)
Mitigations - preventing the peak of maximum capacity of each healthcare provider such as hospitals and Infectious Disease Center; and (2) Suppression - lowering the confirmed cases to low levels through observing social distancing of the entire population, suspension of works and classes in schools and universities, and prohibiting mass gatherings.

In an effort to curb the exponential spread of Covid-19 in the Philippines, the government has implemented the general community quarantine (GHQ) on March 15, 2020. The GHQ mandated the suspension of classes in the National Capital Region, strict observance of social distancing, and calling for the creation of skeletal workforces for offices under the executive branch of the government starting March 16, 2020. The first day of implementation of the GHQ on March 15, 2020 falls on Sunday is seen to be effective in curbing Covid-19 cases. This was due to observed low mobility of people in public places in National Capital Region. The second day of implementation of GCQ seems to be less effective as the number of confirmed Covid-19 cases significantly climbed to 167. This posed the government to expand the GCQ to Enhanced Community Quarantine (ECQ) which took effect last March 17, 2020. The proclamation numbers 929 and 922 series of 2020 and

E-mail address: rldizon@pup.edu.ph. 
Republic Act No. 11332 directed the adoption and implementation of ECQ. The ECQ mandates the following: (1) suspension of work in public and private offices except for the front liners in the government, military personnel, health workers, banking institutions, manufacturing sector, and business process outsourcing; (2) imposed strict home quarantine measures in all households; and (3) suspension of land, air, and water transportations in the entire Luzon.

This study aims to forecast the transmission rate of Covid-19 in NCR, and to investigate the effects of the Enhanced Community Quarantine (ECQ) being implemented by the Philippine Government to prevent the exponential growth of the Corona Virus (Covid-19) in the Philippines.

\section{Materials and methods}

The mathematical modeling plays an important role in epidemiology which serves as a tool in analyzing the spread and control of infectious diseases. It was first used by Volterra which showed that the indefinite coexistence of two or more species limited by the same resources is not possible. According to epidemic model, the infectious disease can be transmitted in two different ways: (1) horizontal transmission which occurs by direct contact between infected and susceptible individuals; and (2) vertical transmission occurs by direct contact between infected materials and susceptible individuals. ${ }^{4}$ Kang and Castillo-Chavez ${ }^{5}$ discussed the Susceptible Infected (SI) model. The population facing a possible transmission of pandemic disease can be effectively captured within Susceptible-Infected framework under the assumptions of multiple possibility of modes of transmission which are horizontal (direct contact) and vertical (indirect contact). The Allee effect alter the net reproduction term due to mating limitation or predator saturations. The Allee Effects is named after scientist W.C. Allee which slow down the rate of population spread and clustering of population. ${ }^{6}$ The SI model is represented by the following notations:

$\frac{d S}{d t}=r S f(N)-\varphi(N) \frac{1}{N} S+\rho r I f(N)-d I$

where, $S$ is the population susceptible in disease, I is the infected population, $\mathrm{N}$ is the total population $(\mathrm{N}=\mathrm{S}+\mathrm{I})$. The first term $r S f(N)$ is the growth of population who are susceptible with Allee effects, the $\varphi(N) \frac{1}{N} S$ is the second term, which is the horizontal transmission (direct contact), $\operatorname{\rho rIf}(N)-d I$ is the third term, which is the vertical transmission $\operatorname{\rho rIf}(N)$ (indirect contact), and the last term $(d I)$ is the additional death due to infections $^{6}$ :

In order to forecast the Covid-19 cases in the NCR, the study used Multi-group Susceptible Infected Recovered Model (SIR) with stage progression, or also known as mathematical models of mixing patterns between age groups. The multi-group SIR is a realistic model for studying the spread of Covid-19 cases, because it take into account the mechanism of its transmission. It incorporate the pattern of mixing among the population, the susceptibility within and between group of population, the probability of transmission per contact. ${ }^{7}$

To implement the Multi-group SIR, this research considered classifying the whole population into three epidemiological classes which are: (1) Susceptible - those population who are disease free based on maximum population capacity and Allee Effect; (2) Infected - those population who are infected by the Covid-19; and (3) Recovered - those population who recovered from Covid-19. These classes are further subdivided into age groups which are: (1) Mobile Workers - those who are exempted in ECQ; (2) Workers under ECQ-those workers who are not exempted in ECQ; (3) Senior Citizen-those population ages 60 years old and above; and (4) Young - those population ages less than 16 years old. This model has the following assumptions and limitations: (1) senior citizen will not be allowed to leave the house for work or to buy necessities since they are more susceptible in the pandemic disease; (2) people who recovered from the infection become immune and cannot become infected the second time around; (3) no available vaccines has

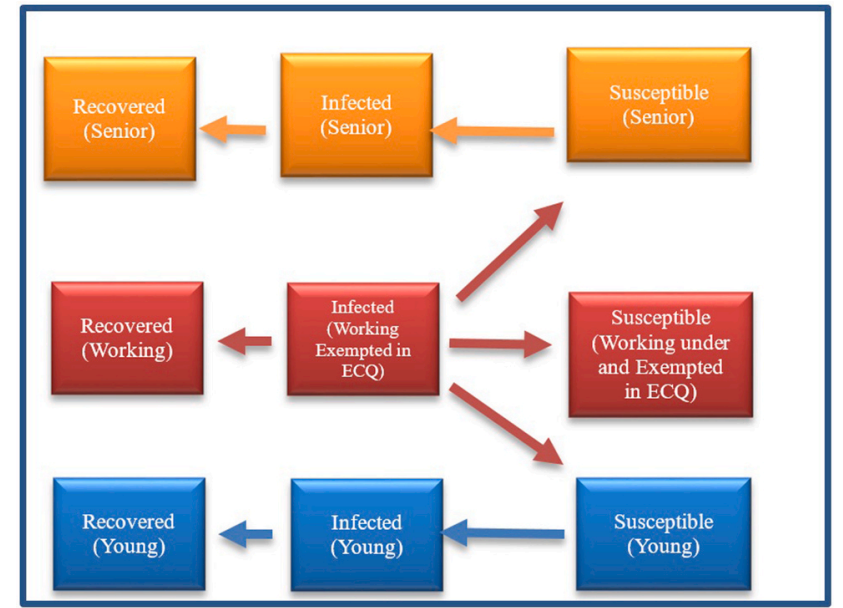

Fig. 1. The mode of transmission of Covid-19 in different age-group.

been developed all around the word, since experiment to create vaccine is still ongoing or vaccines to treat the Covid-19; and (4) in view of data limitations, the chances of transmission to persons who are immunocompromised and with underlying medical conditions with comorbidities can not be estimated using the model. The objective of using this mathematical model of mixing pattern is to determine how each infected individual transmit the infectious disease into the same group, to those working group under quarantine, senior citizen, and young individual. Parameters that have been used in the model are the rate of transmission, period and number of recoveries and fatalities, and probability of transmitting the disease of contacts.

The mathematical model of multi-group SIR model is represented by the following transfer diagram (Fig. 1) which shows the pattern of transmission of Covid-19 disease, which was used in the study. Those working group, who are exempted in the implementation of ECQ, are susceptible to Covid-19 transmission. Once they are infected they maybe able to transmit the disease through direct and indirect to other exempted workers, working population under ECQ, senior citizen and young population age group. The daily total number of population who are infected by the pandemic disease is an aggregation of those who are workers exempted in ECQ, workers under quaratine, senior citizen, and young age group.

The mathematical representation of derivation of those who are Susceptible (S), Infected (I), and Recovered (R) which are measured in days are as follows ${ }^{7}$ :

$i(t)=\lambda_{i}(t) S_{i}(t)-\left(w_{i}+\mu_{i 1}\right) I_{i 1}\left(t_{i}\right)$

$s(t)=-\lambda_{i}(t) S_{i}(t)$

$r(t)=w_{i} I_{i m}$

The $i(t)$ is the change in daily infected people. It was derived by substracting the proportion of susceptible who are infected $\lambda_{i}(t) S_{i}(t)$ and proportion of infected who were expired as represented by $\left(w_{i}+\right.$ $\left.\mu_{i 1}\right) I_{i 1}\left(t_{i}\right)$. The $\mathrm{s}(\mathrm{t})$ is the change in susceptible population. It was derived by determining the proportion of population who are susceptible $\lambda_{i}(t) S_{i}(t)$. The $\mathrm{r}(\mathrm{t})$ is the change in recovered from the disease. It was derived by determining the proportion of population who recovered. The $\lambda_{i}(t)$ is the rate of disease transmission from infected people in working group to same age group, senior citizen group, and young group. It is derived using the following formula:

$\lambda_{i}(t)=\gamma(t)\left(P_{i j}\right)\left(\frac{I_{2}}{N_{2}}\right)$

where, $\gamma(t)$ is the number of contact per day, $\left(P_{i j}\right)$ is the Probability of 

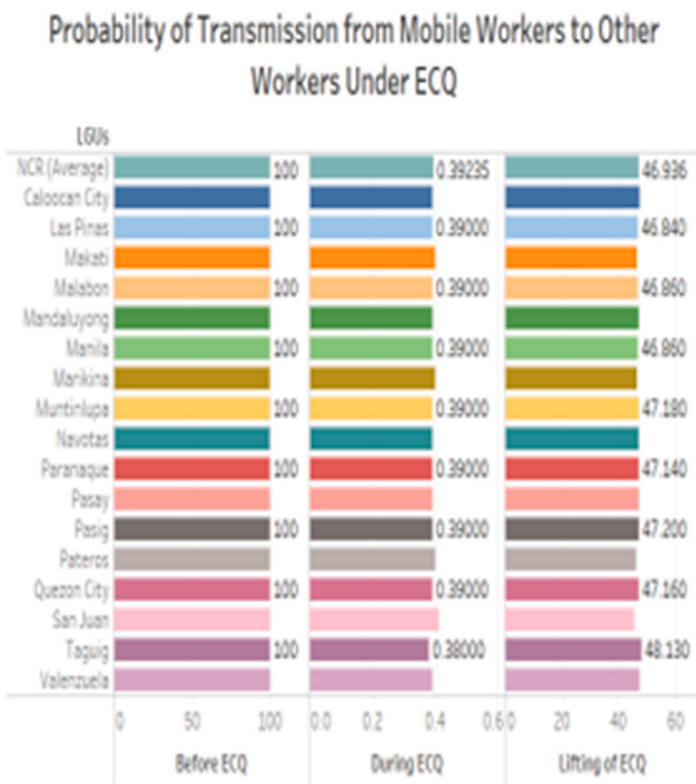

\section{Probability of Transmission from Mobile Workers to Senior} Citizen

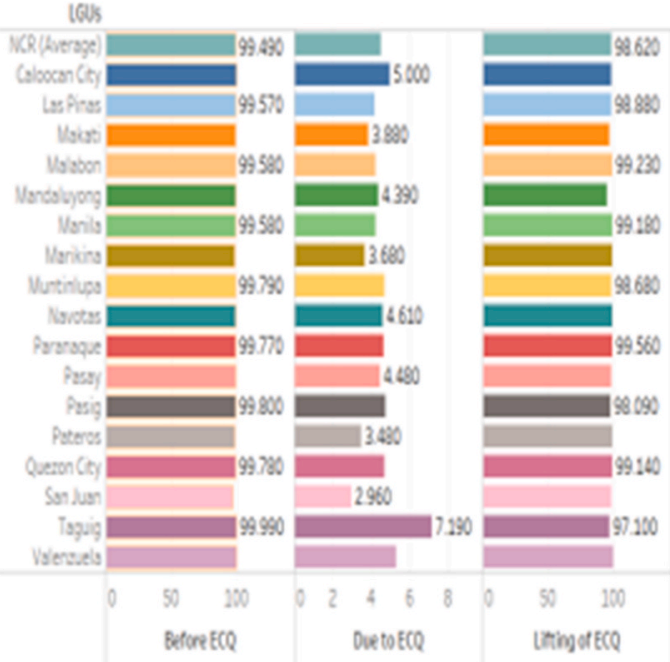

\section{Probability of Transmission from Mobile Workers to Young Citizen}
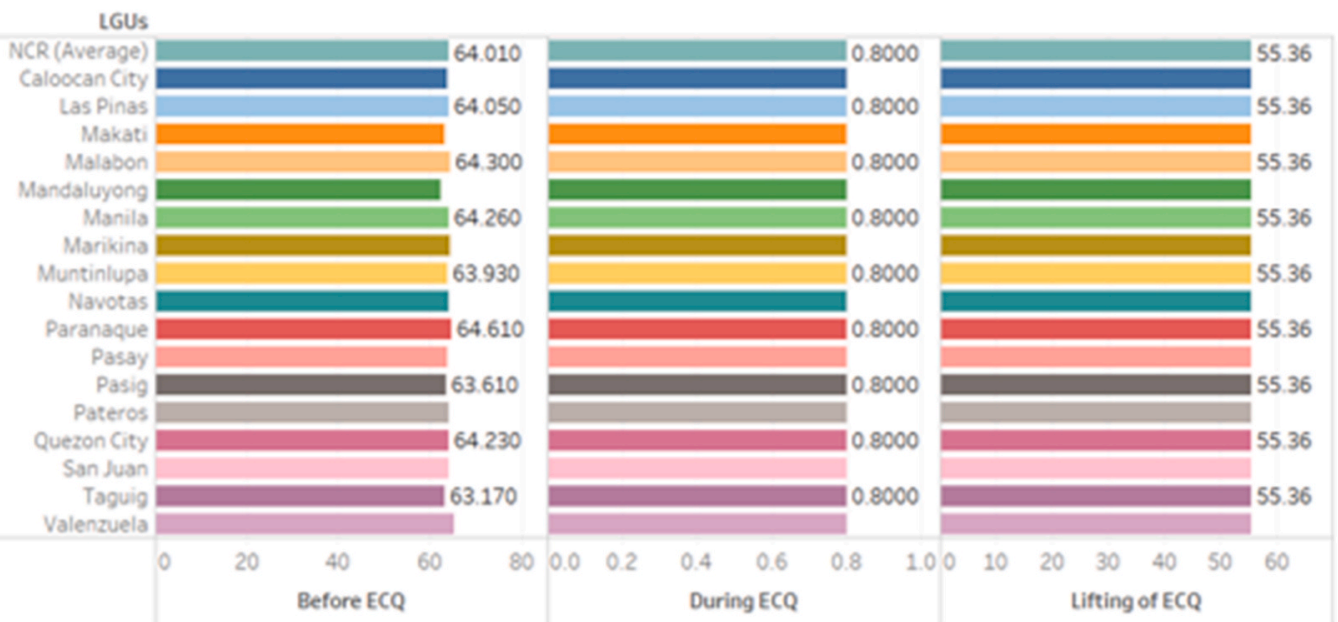

Fig. 2. The Probability of transmission from workers to other workers, to senior citizen,and to young citizen.

disease transmission per day using the Poisson Distribution, $\left(\frac{I_{2}}{N_{2}}\right)$ is the proportion of contacts who are infected. The Poisson distribution is given by:

$P_{i j}=1-e^{-\varsigma T_{i j}}$

where Tij is the average duration of a contact of an individual from working group to same age group, senior citizen, and young group. The $\varsigma$ is the normal mixing pattern. The normal mixing pattern reflects the preferential mixing between the ages on a normal day in the absence of disease. In this study, the normal mixing used is the per day contact of those total susceptible working age to the same group, to senior citizen, and to young group.

The number of infected cases, recovered, and fatal were taken from Department of Health ${ }^{15}$ and Philippine Statistical Authority. ${ }^{16}$

\section{Results}

This research simulated two different scenarios of implementation of Enhanced Community Quarantine which are composed of (1): Effects of ECQ; and (2) Lifting of ECQ on April 14, 2020.

In the absence of ECQ the probability (chances) of transmitting Covid-19 disease in NCR across mobile workers is at 100\%, from workers to senior citizen is $99.49 \%$ and to the young group is $64.01 \%$ (Fig. 2). Based on the data from March 17 to April 19, 2020, with continuous implementation of ECQ, the chances of transmitting this pandemic disease from mobile workers to workers under ECQ dropped to an average of $0.39 \%$, to $4.50 \%$ for senior citizen, and $0.8 \%$ for the young age group, across all LGUs in NCR. The supposed partial lifting of ECQ last April 14, 2020 was perceived to result in an increase in transmission by $46.94 \%$ (workers under ECQ), $98.62 \%$ (senior citizen) and $55.36 \%$ (young).

By the end of April 2020, the Covid cases in NCR is forecasted to reach up to 7000 amid ECQ. This is much lower compared to the predicted 900,000 cases if the ECQ was prematurely lifted on April 14, 

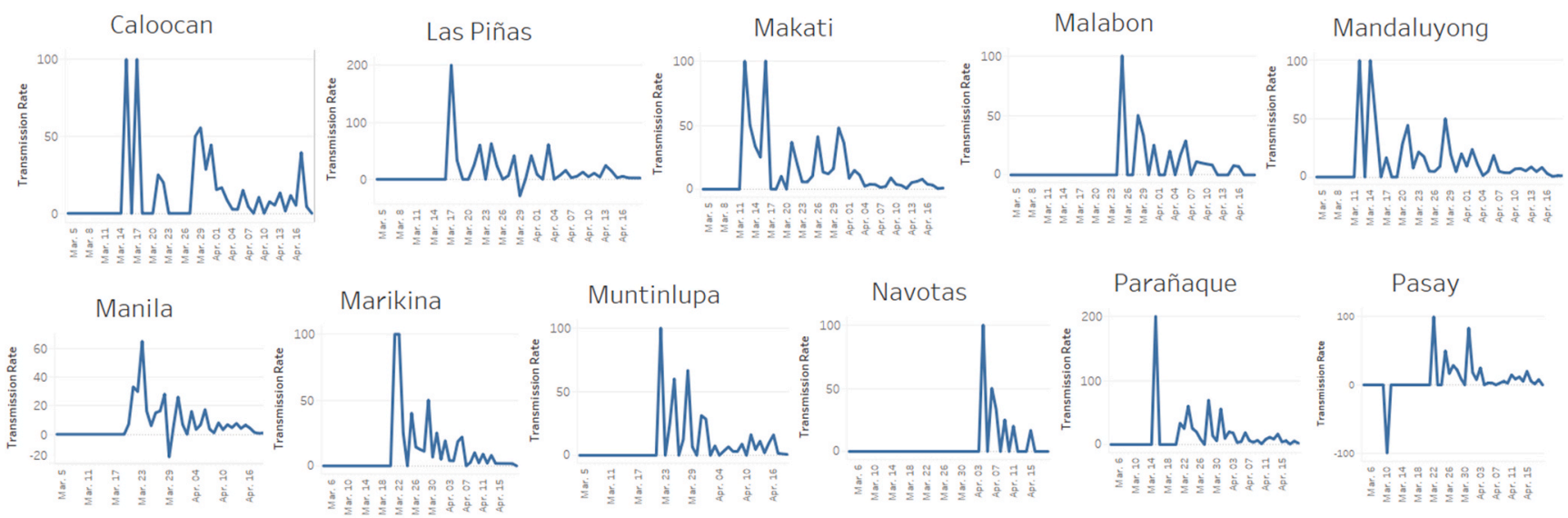

Marikina

Muntinlupa

Navotas
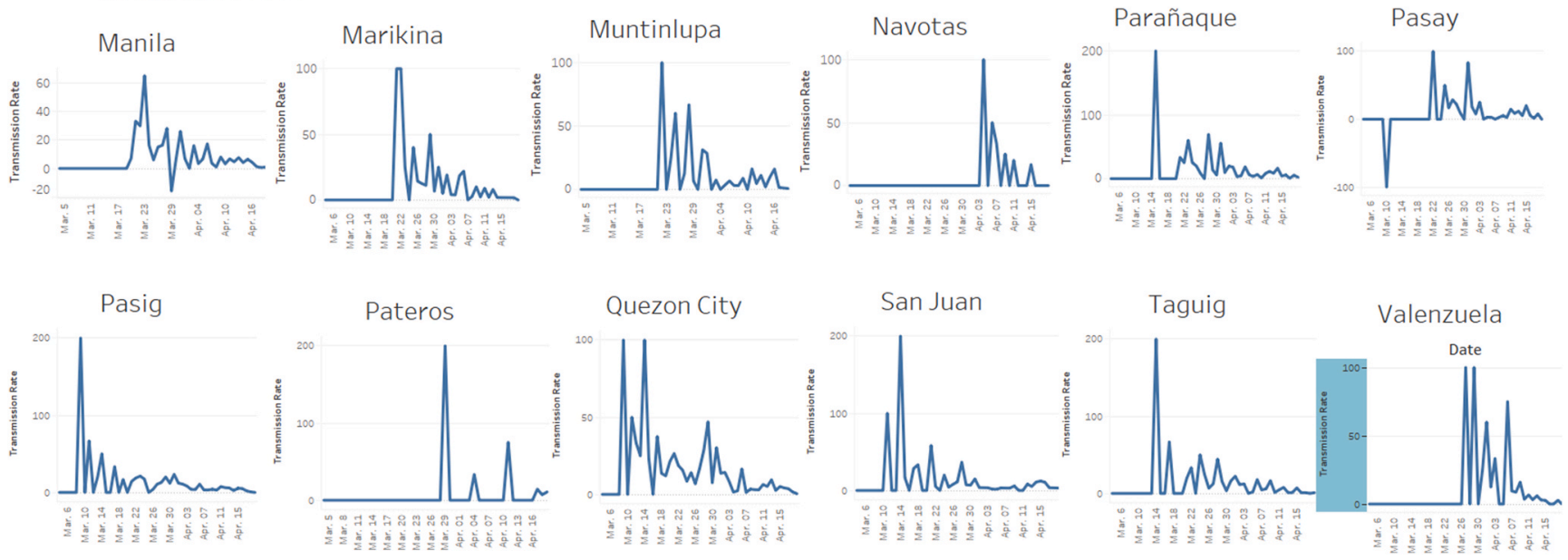

Fig. 3. The Covid-19 transmission rate in the National Capital Region, Philippines.

2020. The per density transmission rate of Covid-19 cases in various 17 Local Government Units (LGUs) in the National Capital Regions is shown in Fig. 3. Most of the LGUs is nearing towards consistent zero transmission rate (i.e. nearing flattening the curve) of Covid-19 in the group of mobile workers. A possible explanation of this is, with more than five weeks of ECQ implementation, workers have somehow adopted to the "new normal." That is, the habit of regular wearing of protective masks, ensuring physical or social distancing and frequent handwashing or disinfecting of hands. Among LGUs, Caloocan showed a sluggish movement of transmission rate where associated with continues mobility of people under quarantine which affects the movement of transmission to be more or less concentrated into zero.

\section{Discussions}

Table 1 shows the estimated peak period of transmission of Covid-19 Cases in different Local Government Units in the National Capital Region based on mathematical model of heterogenous pattern of mixing of different groups of individual. Analyzing the pattern of the number of
COVID-19 cases per group classification, It was observed that the curve for mobile workers are expected to flatten first, and then followed by the simultaneous flattening of the curves of the workers under ECQ, senior citizens and the young age group. Interestingly, Quezon City will have a different order of flattening of the curves: those of the mobile workers first to reach flattening of the curve, followed by the young age group, the workers under ECQ and then by the senior citizens. In the City of Manila, the sequence of flattening of the curves of the four groups is: the mobile workers first to attain flattening of the curve, followed by senior citizens group, the young age group and then the workers under ECQ.

The result of study revealed that after April 30, 2020, most of the LGUs in NCR still need at least two more weeks of ECQ to completely attain flattening of the curve (i.e., when all the four groups cited above have reached plateau in their respective curves). Based on the model, LGUs which are expected to completely flatten the curve on the second week of May are Las Piñas, Makati, Malabon, Mandaluyong, Manila, Muntinlupa, Marikina, Pasay, Pasig, Pateros, Quezon City, Taguig, and Valenzuela. Meanwhile, Caloocan, Navotas, Parañaque, and San Juan are predicted to attain the flattening of the curve in the last week of May.

Table 1

The estimated peak period of transmission of Covid-19 of different local government units in the National Capital Region, Philippines.

\begin{tabular}{|c|c|c|c|c|c|}
\hline LGUs & Mobile Peak Period & Workers Peak Period & Senior Peak Period & Young Peak Period & Disease Free Period \\
\hline Caloocan & First Week of June & Second Week of June & Third Week of May & Third Week of May & First Week of July \\
\hline Las Piñas & First week of May & Second Week of May & Second Week of May & Second Week of May & First Week of July \\
\hline Makati & First week of May & Second Week of May & Second Week of May & Second Week of May & Last Week of June \\
\hline Malabon & Third Week of April & Last Week of April & & Second Week of May & Third Week of May \\
\hline Mandaluyong & First week of May & Second Week of May & Second Week of May & Second Week of May & Last Week of May \\
\hline Manila & First week of May & Second Week of May & Second Week of May & Second Week of May & Second Week of June \\
\hline Marikina & Last Week of April & Second Week of May & Second Week of May & First Week of May & Third Week of June \\
\hline Muntinlupa & First week of May & Second Week of May & Second Week of May & Second Week of May & Second Week of July \\
\hline Navotas & First week of May & Second Week of May & Second Week of May & Last Week of May & Third Week of May \\
\hline Parañaque & Second Week of May & Last Week of April & Last Week of May & Last Week of May & Third Week of June \\
\hline Pasay & Last Week of April & Second Week of May & Second Week of May & Second Week of May & Second Week of June \\
\hline Pasig & Last Week of April & Second Week of May & Second Week of May & Second Week of May & First Week of August \\
\hline Pateros & Second Week of May & First Week of May & Second Week of May & First Week of May & Third Week of May \\
\hline Quezon City & First week of May & Second Week of May & Second Week of May & Second Week of May & Second Week of July \\
\hline San Juan & Second Week of May & First Week of May & Third Week of May & Third Week of May & Last Week of June \\
\hline Taguig & First week of May & Second Week of May & Second Week of May & Second Week of May & First Week of July \\
\hline Valenzuela & First week of May & Second Week of May & Second Week of May & Second Week of May & Third Week of May \\
\hline
\end{tabular}


After the period of a flattened curve, there is the recovery period, which ranges from the shortest of 7 days for workers and the young age group to a maximum of 45 days for the senior citizens group. Factoring this recovery period, LGUs that attained a flattening of the curve by the second week of May will reach a state of equilibrium (i.e., the state of zero transmission rate and that the number of cases coincides with the number of recovered cases and number of deaths) in the last week of June; while those reaching a flattening of the curve in the end of May are forecasted to attain equilbrium by the end of July this year. However, because of a very low recovery rate that is associated with long recovery period, the City of Pasig, based on the model, will realize an equilibrium state in the first week of August. An additional quarantine period of 14 days is usually required of a recovered patient in order to observe whether a relapse has occurred. Thus, factoring this into the scenario, all of the LGUs in NCR shall be considered cleared of the disease and the community quarantine may be lifted by the third or fourth week of August.

\section{Conclusions}

Based on the result of this finding, the NCR needs extension of two more weeks of Enhanced Community Quarantine and per LGU cases of lifting thereafter the second week of May 2020. Those baranggay which still have increasing cases of transmission should remain in ECQ. After lifting ECQ, LGUs should still follow the protocol to prevent the further spread of Covid-19 such as observing social distances, avoid mass gathering, gradual opening of operations of certain essential company, and wearing masks when in close contact with other people.

The equilibrium between recovery rate, fatality rate, heterogonous mixing pattern of transmission rate in different age groups should take into account as the basis of lifting the ECQ. The government should continue the work-from-home scheme at the end of May 2020 and observed skeletal forces until July 2020 in the National Capital Region, so as to further prevent the spread of disease since there are still possibility of transmission due to unrecovered patients that may still transmit the disease to other people. Consider lifting the ECQ in some areas outside NCR which have zero transmission rate for the past 15 days and wide household distance. This would allow the economy to lessen the impact of the government intervenion in curbing the spread of pandemic disease in the local and national economy. Implement stringent ECQ scheme by creating a law that penalizes those who will violate quarantine rules.

The government should provide ample temporary shelter for the health service workers such as doctors, nurses, and other medical staff, and those who are working in the Armed Forces of the Philippines and Philippine National Police to limit their mobilization since they are more susceptible in the Covid-19 cases.

\section{Funding}

No Funding.

\section{Ethical approval}

Not required.

\section{Declaration of competing interest}

None of the author have conflict of interest to report.

\section{Acknowledgements}

The author would like to acknowledge the invaluable inputs and help of the following Officials of the PUP and individuals: PUP President Dr. Manuel M. Muhi, Executive Vice President Alberto C. Guillo, VPAA Emanuel C. De Guzman, VP for Research Extension \& Development Anna Ruby P. Gapasin, Assistant to the VP RED Tomas O. Testor, Assistant to the VPAA Edelyn M. Mariano, OU Executive Director Carmencita L. Castolo, Dra. Liza T Yanes of PUP Medical Department, Dir. Angie Borican, Dr. Philip Randy P. Tuaño of Ateneo De Manila University Department of Economics, Mr. Nanie Gonzales, and Dr. Melcah P. Monsura. This current study is an expansion of the previous research on assessment of the effects of Enhanced Community Quarantine on curbing the spread of Covid-19 published last March 19, 2020.

\section{References}

1 Guo Y, Qing-Dong C, Hong Z, et al. The origin, transmission and clinical therapies on coronavirus disease 2019 (COVID-19) outbreak- an update on the status. Mil Med Res. 2020;7:11. Retrieved from https://mmrjournal.biomedcentral. com/articles/10.1186/s40779-020-00240-0\#citeas last. Accessed March 23, 2020.

2 World Health Organization. Retrieved from https://www.who. int/docs/defaultsource/coronaviruse/situation-reports/20200322-sitrep-62-covid19. pdf?sfvrsn =f7764c46_2 last; 2020. Accessed March 24, 2020.

3 Pellis, N. Ferguson, \& Frase, C. Threshold Parameters for a Model of Epidemic Spread Among Household and Workplaces. J Roy Soc Interface, 6(4) https://doi.org/10. 1098/rsif.2008.0493.

4 Naji R, Hussien R. The dynamic of epidemic model with two type of infectious diseases and vertical transmission. J Appl Math. 2016;16:16.

5 Kang, Y., \& Catillo-Chavez, C. Dynamics of SI models with both horizontal and vertical transmissions as well as allee effects. Math Biosci. Doi: 10.1016/j.mbs.2013.12.006.

6 Greene Cm. Allee effects. Encycl Ecol. 2008:123-127. https://doi.org/10.1016/B978008045405-4.00639-X.

7 Del Valle SY, Hyman JM, Chitnis N. Mathematical models of contact patterns between age groups for predicting the spread of infectious diseases. Math Biosci Eng: MBE. 2013;10(5-6):1475-1497. https://doi.org/10.3934/mbe.2013.10.1475.

15 Department of Health. Retrived from https://ncovtracker.doh.gov.ph/last; 2020. Accessed March 24, 2020.

16 Philippine Statistical Authority. Retrieved from https://psa.gov.ph /content/2017-annual-survey-phili ppi ne -business-and-industry-aspbi-financial-and-insurance-0 last; 2020. Accessed March 24,2020 . 